\title{
Torsion of a fallopian tube: a rare cause of lower abdominal pain in a female of reproductive age
}

\author{
Janitha R Costa ${ }^{1}$, James Moohan ${ }^{1}$ \\ Sri Lanka Journal of Obstetrics and Gynaecology 2011; 33: 63-64
}

\begin{abstract}
Isolated unilateral torsion of a fallopian tube is an infrequent but significant cause of acute lower abdominal pain in a female of reproductive age. We present a literature review and the case of a 41-year old lady who presented with sudden onset right-sided lower abdominal pain radiating to right thigh. Clinical examination revealed tenderness in the right lower quadrant of the abdomen and a tender right adnexal mass was noted on vaginal examination. Transvaginal scan revealed an elongated, multiloculated right adnexal mass and a normal left ovary. No free fluid or ascites were noted. Torsion of a right hydrosalpinx was suspected and an emergency laparoscopy was performed which confirmed the diagnosis and a right salpingectomy was carried out. Isolated torsion of a fallopian tube should be considered in the differential diagnosis of lower abdominal pain in any female of reproductive age, particularly when she has a history of tubal sterilisation, oophorectomy and/or pelvic adhesive disease.
\end{abstract}

Key words: torsion, fallopian tube, hydrosalpinx, adnexal mass, salpingectomy.

\section{Introduction}

Isolated torsion of the fallopian tube was first reported in 1890 by Bland-Sutton ${ }^{1}$. Since this first description, torsion of a normal as well as abnormal tube has been reported ${ }^{1-7}$. The overall incident has been reported as 1 in 1.5 million women ${ }^{2}$, with preoperative diagnosis being made in less than $20 \%$ of reported cases $^{3}$ and laparoscopy remains the gold standard for diagnosis and treatment.

\section{Case report}

A 41-year old parous lady presented on day 21 of her menstrual cycle with a 24 hour history of sudden

\footnotetext{
${ }^{1}$ Department of Obstetrics and Gynaecology, Altnagelvin Area Hospital, Londonderry BT47 6SB.

Correspondence: Janitha Costa

E-mail: janitharuwanthi@yahoo.co.uk
}

onset right sided lower abdominal pain which radiated to her right thigh. The pain did not respond to opioid analgesia. Of note in her medical history were the fact that she had undergone a right oophorectomy 7 years previously and a laparoscopic sterilisation using filshie clips, 5 years prior to the presentation. Clinical examination elicited tenderness in the right lower quadrant of the abdomen but there were no evidence of peritonism. Bimanual pelvic examination revealed a tender mass in the right adnexa. Urinary hCG was negative, white blood cell count was normal and CRP was within normal limits. Transvaginal ultrasound examination (sonosite $4 \mathrm{Mhz}$ ) showed an elongated, multiloculated mass measuring $7.8 \mathrm{~cm} \times 5.26 \mathrm{~cm}$ in the right adnexal region (Figure 1)

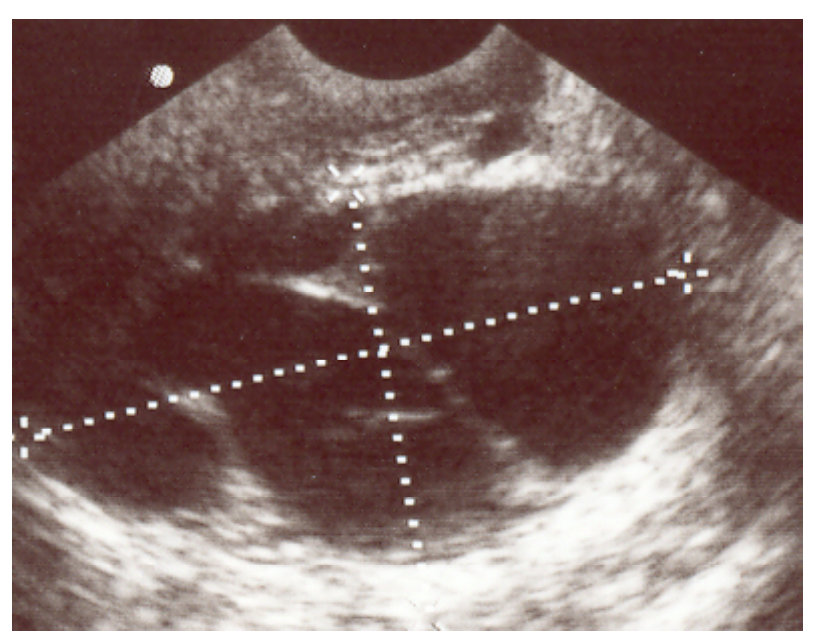

Figure 1. Transvaginal ultrasound image of elongated, distended fallopian tube $(7.8 \mathrm{~cm} \times 5.6 \mathrm{~cm})$.

There was no ascites or free fluid and the left ovary appeared normal. Torsion of a right hydrosalpinx was suspected and an emergency laparoscopy was performed. This confirmed torsion of a right-sided hydrosalpinx with the fulcrum of torsion being the filshie clip. A right salpingectomy was performed and the post-op course was uneventful with patient being discharged the next morning.

Histopathology report confirmed dilated fallopian tube with haemorrhagic patches and ischemic changes. 


\section{Discussion}

Isolated tubal torsion is a rare but significant cause of acute lower abdominal pain in a female 4 . The diagnosis is infrequently made preoperatively due to lack of pathognomonic symptoms and clinical findings. Although the exact cause for tubal torsion is unknown, torsion of an intact, healthy tube is unusual $^{6}$. Yourself et $a l^{7}$ classified factors that could influence the occurrence of tubal torsion into two groups: intrinsic and extrinsic. Intrinsic causes include congenital abnormalities such as excessive length of tube or a spiral course, hydrosalpinx, haematosalpinx, tubal neoplasm and prior surgery particularly tubal sterilization. Ovarian and paratubal masses, pregnancy, adhesions, pelvic congestion and sudden body movements are listed as extrinsic causes. All these factors contribute to the development of tubal torsion by providing a point of reference (fulcrum) around which the tube can twist. Our patient had previously undergone tubal sterilisation using filshie clips and right oophorectomy which allowed the tube more freedom to rotate and the filshie clip which acted as the fulcrum. Symptoms of tubal torsion include the sudden onset of unilateral lower abdominal pain radiating to the groin or anterior/lateral aspect of thigh $^{5}$ as in this case. The condition is frequently associated with nausea and vomiting and occasionally urinary symptoms such as frequency and urgency with voiding difficulties ${ }^{5}$ and all these symptoms were absent in our patient. The patient is usually haemodynamically stable with signs of peritonism often being absent at presentation and only being manifest with the necrosis of the tube. Bimanual pelvic examination may reveal a tender adnexal mass associated with cervical excitation while laboratory studies are usually within the normal range as in this case. Pelvic ultrasound may reveal an elongated cystic mass with variable septations and scattered internal echoes as seen in (Figure 1). Colour Doppler Transvaginal Sonography may show a high impedance waveform with reversal of diastolic flow in the affected tube ${ }^{3,5}$ due to obstruction of the blood supply in the mesosalpinx. When evaluating a female patient with acute lower abdominal pain, tubal torsion may not be high in the list of differential diagnosis with diagnosis often being made at the time of surgery. Laparoscopy remains the gold standard for the diagnosis and treatment as the early diagnosis may allow detorsion/salvage of the tube. In our case, the right oophorectomy and tubal sterilization put her at increased risk of tubal torsion. In conjunction with the clinical findings and ultrasound examination, this enabled us to make a preoperative diagnosis of torsion of a right hydrosalpinx. Although laparoscopic tubal detorsion is preferred over salpingectomy in patients of reproductive age in the absence of ischaemic damage, an abnormal tube or the suspicion of malignancy, salpingectomy is the treatment of choice if the tube is gangrenous, there is a suspected adnexal malignancy or tube is diseased as in this case. A further indication for salpingectomy is if patient has completed family as in this case.

\section{Conclusion}

Fallopian tube torsion is a rare but significant cause for acute lower abdominal pain in women of reproductive age. Clinicians should consider tubal torsion in the differential diagnosis in women who exhibit predisposing factors.

\section{References}

1. Bland-Sutton T. Salpingitis and some of its effects. Lancet 1890; 2: 1146.

2. Hansen O. Isolated torsion of the fallopian tube. Acta Obstet Gynaecol Scand 1970; 49: 3.

3. Trevor D, Lineberry, Rodriguez H. Isolated torsion of the fallopian tube in an adolescent. Journal of Paediatric Adolescent Gynaecology 2000; 13: 135-8.

4. Diaa E, Rizk E, Lakshminarasimha B. Torsion of the fallopian tube in an adolescent female. Journal of Paediatric Adolescent Gynaecology 2002; 15: 159-61.

5. Krissi H, Shalev J, Bar-Hava I. Fallopian tube torsionlaparoscopic evaluation and treatment. JABFP 2001; 14(4): 274-7.

6. Krissi H, Orvito R. Torsion of a fallopian tube following a pomeroy tubal ligation. European Journal Obstet and Gynaecol 1997; 72: 107-9.

7. Youssef AF, Fayad MM, Shafeek MA. Torsion of the fallopian tube. A clinico-pathological study. Acta Obstet Gynae Scand 1962; 41: 292-309.

8. Sozen, Kadako I, Fleischman R, et al. Diagnosis and laparoscopic management of a fallopian tube torsion following Irving tubal sterilisation. Surgical Endoscopy Jan 2002; 16(1): 217. 